\title{
Taller de Inducción para Aplicación de los Fondos Concursables de Proyectos de Innovación de UNAN-Managua 2015
}

El día 11 de agosto del 2015 se llevó a cabo el Taller de Inducción para la Aplicación de los Fondos Concursables de Proyectos de Innovación de UNAN Managua 2015 en el auditorio de la Biblioteca Salomón de la Selva.

\section{Objetivos}

Los objetivos de dicho taller fueron los siguientes:

$\checkmark$ Publicar la Segunda Convocatoria de Proyectos de Innovación de UNAN Managua 2015

$\checkmark$ Motivar a los estudiantes a aplicar en los Fondos Concursables de Proyectos de Innovación de UNAN Managua 2015 y en la IX edición del Premio Nacional a la Innovación.

$\checkmark$ Promover la Red de estudiantes innovadores y emprendedores de UNAN Managua y Red de docentes innovadores y emprendedores de UNAN Managua.

$\checkmark$ Incentivar a los estudiantes en los procesos de fondos competitivos y su impacto en la calidad profesional mediante experiencias de jóvenes innovadores y emprendedores de éxitos.

$\checkmark$ Crear compromisos en estudiantes y docentes participantes siendo facilitadores en divulgar la información recibida, replicar en sus facultades los conocimientos adquiridos y aplicar a los fondos de innovación.

\section{Programa}

\section{Bloque}

Se inició con la inscripción de los participantes, se les entregó una carpeta enumerada del número 1 al 5 para la debida organización en las mesas de trabajo.

Seguido con las palabras del MSc. Javier Pichardo, Vicerrector de Investigación UNANManagua, hace énfasis en que la UNAN Managua está enfocada en fortalecer los procesos de innovación. 


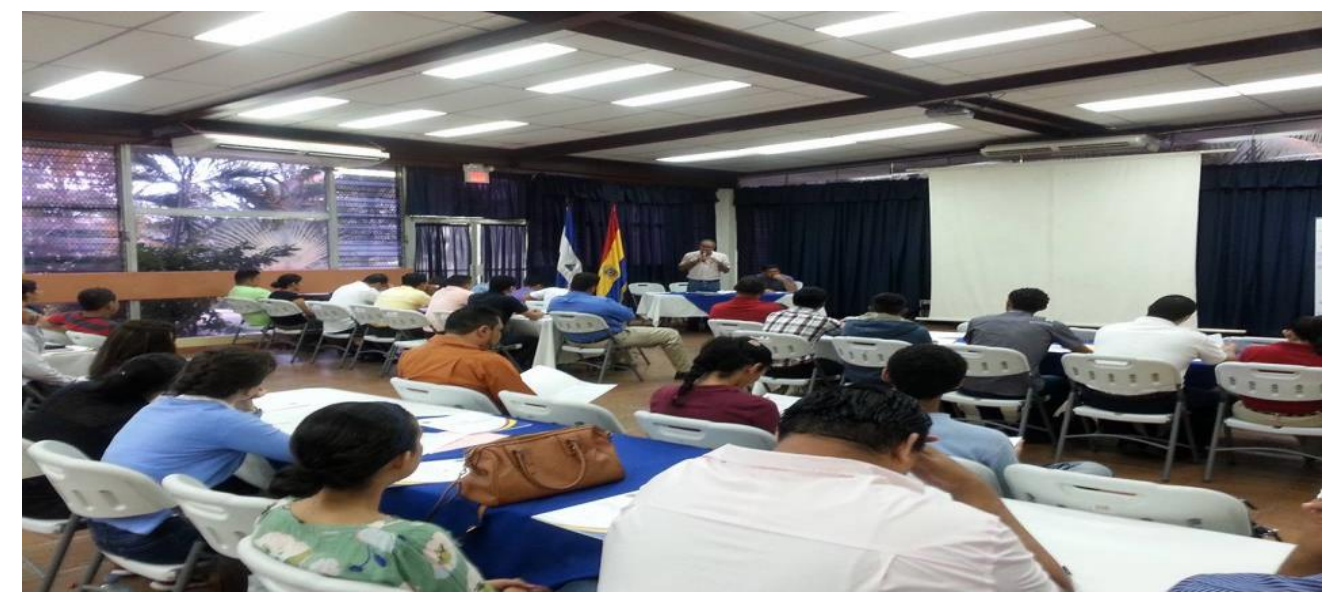

Figura 1: MSc. Javier Pichardo Vicerrector de Investigación UNAN- Managua

Después el MSc. César Pereira Morales, Coordinador del Área de Innovación de UNANManagua explicó los objetivos del taller de inducción y presentó a los miembros de la Comisión de Innovación ante los participantes.

Después la Maestra Violeta Gago, Asistente del Área de Innovación, realizó una presentación sobre la Segunda Convocatoria de Proyectos de Innovación mediante el mecanismo de Fondos Concursables 2015 de la UNAN - Managua. Destacando los siguientes aspectos:

1. El propósito de esta convocatoria está orientado a la entrega de recursos económicos a todos los estudiantes de grado de la UNAN - Managua que presenten propuestas de proyectos innovadores enmarcados en las áreas de innovación establecidas por el Área de Innovación de la Dirección de Investigación de Grado y de pertinencia para resolver problemas del país y en particular para la educación superior. Este fondo es para promover la creación de prototipos funcionales innovadores. La convocatoria se cierra el 21 de agosto.

2. La profesora también explicó los siguientes elementos:

$\checkmark$ Objetivos de la convocatoria

$\checkmark$ Bases generales de la convocatoria

$\checkmark$ Procedimiento para aplicar a la convocatoria

$\checkmark$ Evaluación de los proyectos

$\checkmark$ Financiamiento

$\checkmark$ Líneas de innovación

$\checkmark$ Formalización del financiamiento

$\checkmark$ Calendario 
Seguido se realiza la presentación sobre experiencia de éxito del Lic. Julio Baltodano empresario joven y gerente de su propia empresa llamada Textil Iko Imagen.

Iko Imagen, una pequeña empresa que elabora productos novedosos, tales como bolsos para traslados de valores y mochilas escolares con diseños originales de la empresa. Esto les ha permitido hacerse de un nombre en corto tiempo entre el segmento de las agencias publicitarias e instituciones bancarias, los cuales se han convertido en sus mejores clientes

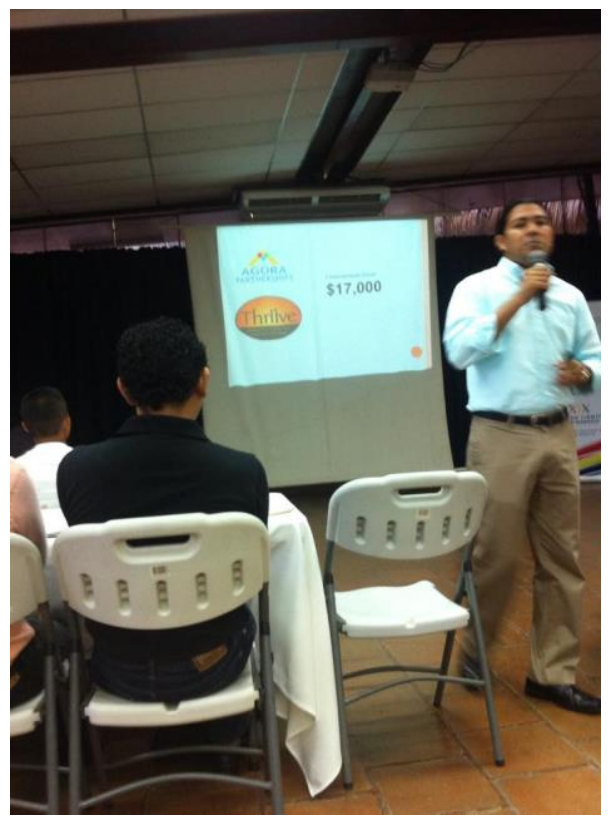

Figura 2: Lic. Julio Baltodano. Gerente de Iko Imagen

Después la Secretaria Ejecutiva del CONICYT, MSc. Guadalupe Martínez presente las siguientes convocatorias:

\section{Edición del Premio Nacional a la Innovación}

El Premio Nacional a la Innovación tiene como objetivo fomentar una cultura científica y/o de innovación, impulsando la colaboración entre el sector académico y el sector empresarial e incentivando y proyectando la puesta en marcha de proyectos innovadores que contribuyan a resolver problemas planteados en las estrategias del Plan Nacional de Desarrollo Humano (20122016), generando beneficios directos a la población. 
Este Premio crea un espacio muy importante para los participantes, permitiéndoles presentar sus proyectos innovadores y competir para obtener un reconocimiento nacional además de realizar las gestiones necesarias para la divulgación internacional de sus esfuerzos.

\section{Convocatoria Reconocimiento a la trayectoria científica}

Reconocimiento a una vida dedicada a la Ciencia y la Tecnología, el cual será otorgado a personas que hayan dedicado su vida a la generación de conocimientos, la difusión de las ciencias, la formación de recursos humanos, la innovación tecnológica o la aplicación de la ciencia y la tecnología al desarrollo humano.

Reconocimiento al Logro Científico, será otorgado a la persona que en el transcurso de los dos últimos años haya alcanzado la mayor productividad e impacto en la generación de conocimiento.

Reconocimiento al Científico Novel, será otorgado al joven científico que en los dos últimos años haya alcanzado el mayor impacto en el conocimiento generado.

Reconocimiento a la Promesa Científica, será otorgado a los estudiantes de la educación primaria, secundaria o técnica que presenten el proyecto de investigación o científico de mayor originalidad y potencial beneficio para el desarrollo humano del país.

\section{Concurso Juvenil Aplicaciones Móviles}

Dirigida a jóvenes menores de 25 años, la temática es libre, pueden participar individual o en pareja, se evalúa funcionalidad y originalidad. Menciono la Maestra Martínez, que todas las convocatorias se cierran el 01 de septiembre.
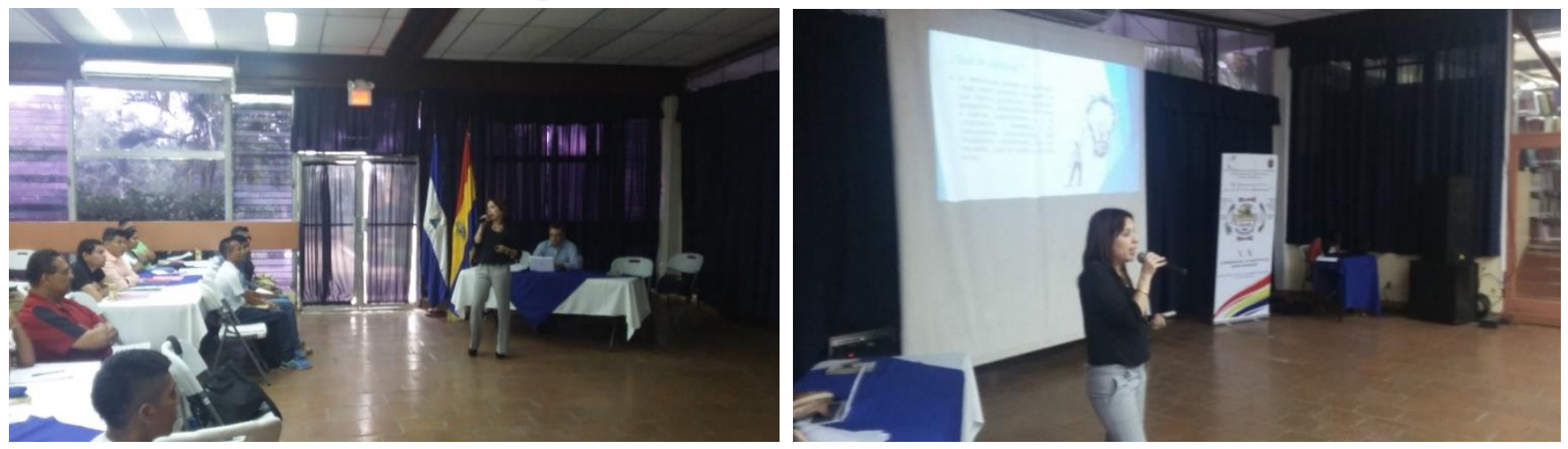

Figura 3 y 4: MSc. Guadalupe Martínez - CONICYT 
Después el MSc. Ernesto Gómez realiza una presentación sobre la Red de Jóvenes Investigadores, Innovadores y Emprendedores de UNAN Managua, el objetivo fue motivar a los estudiantes a integrarse a la red y ser parte de las actividades de innovación y emprendimiento de la UNAN - Managua. Se les entregó un formato de inscripción en físico para los estudiantes que quisieran formar parte de la red.

La MSc. Leyla Argüello realiza una presentación sobre la Red de Docentes Investigadores, Innovadores y Emprendedores de UNAN Managua. También en la dirección de motivar a los docentes presente a formar parte de esta red y fortalecer los procesos de innovación y emprendimiento.

\section{Bloque}

La metodología de desarrollo del segundo bloque fue basada en un enfoque "Aprender haciendo", los participantes se organizaron en 5 mesas de trabajo por línea de innovación, cada participante se agrupó en la mesa correspondiente al número asignado en la carpeta entregada al momento de la inscripción. Cada mesa estaba conformada por 8 estudiantes y 4 docentes.

$\checkmark$ Mesa 1: Innovación en Medio Ambiente y Cambio Climáticos.

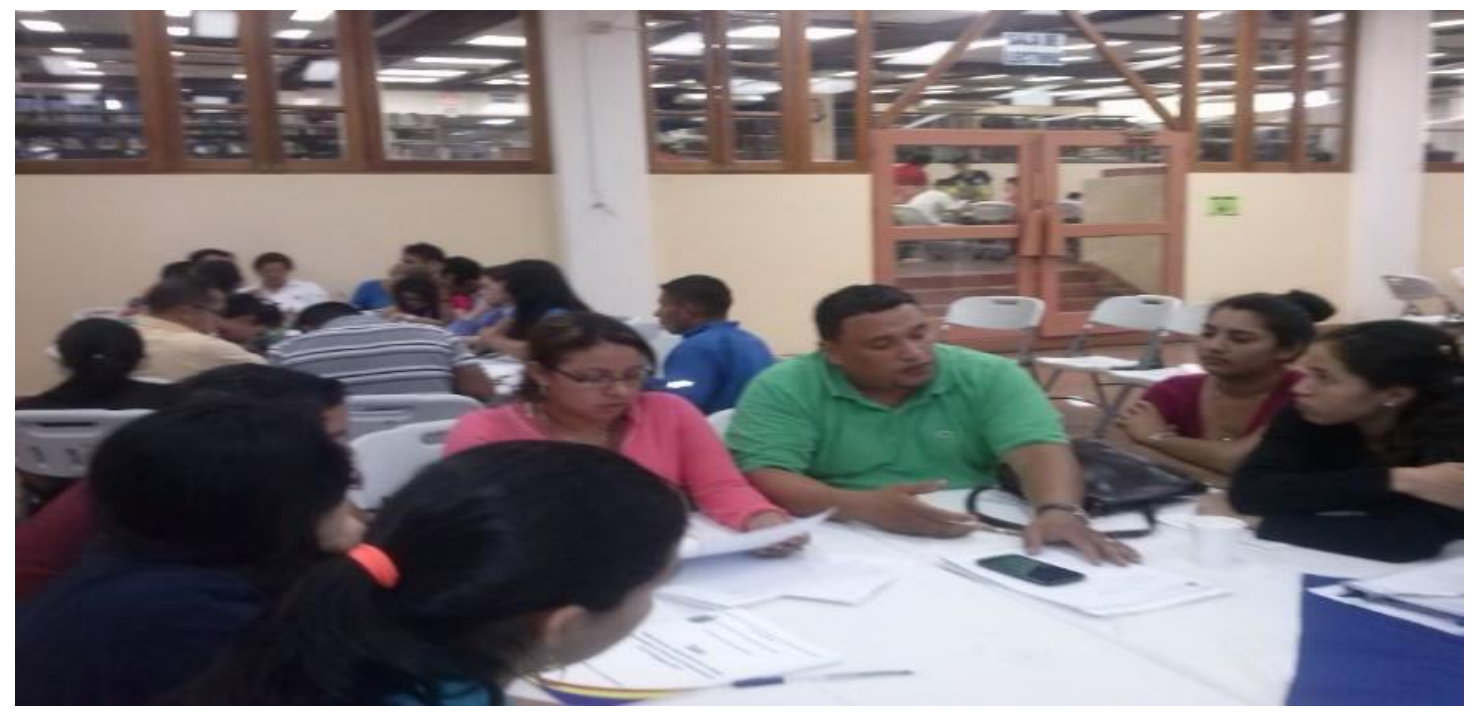

Figura 4. Coordinadores: MSc. Roberto Cano/ MSc. Xochil Zambrana 


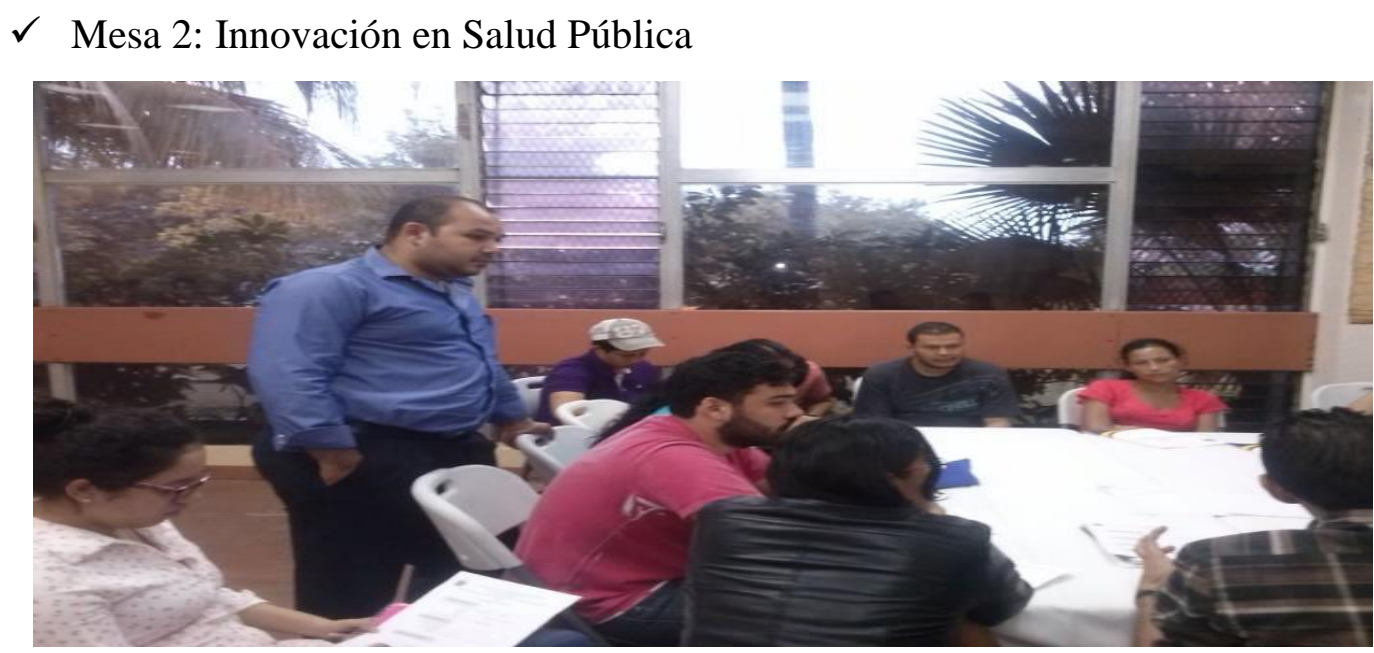

Figura 5. Coordinadores: Dr. Wilbert López/ Dr. Teodoro Tercero

$\checkmark$ Mesa 3: Innovación en Tecnologías de la Información - TIC

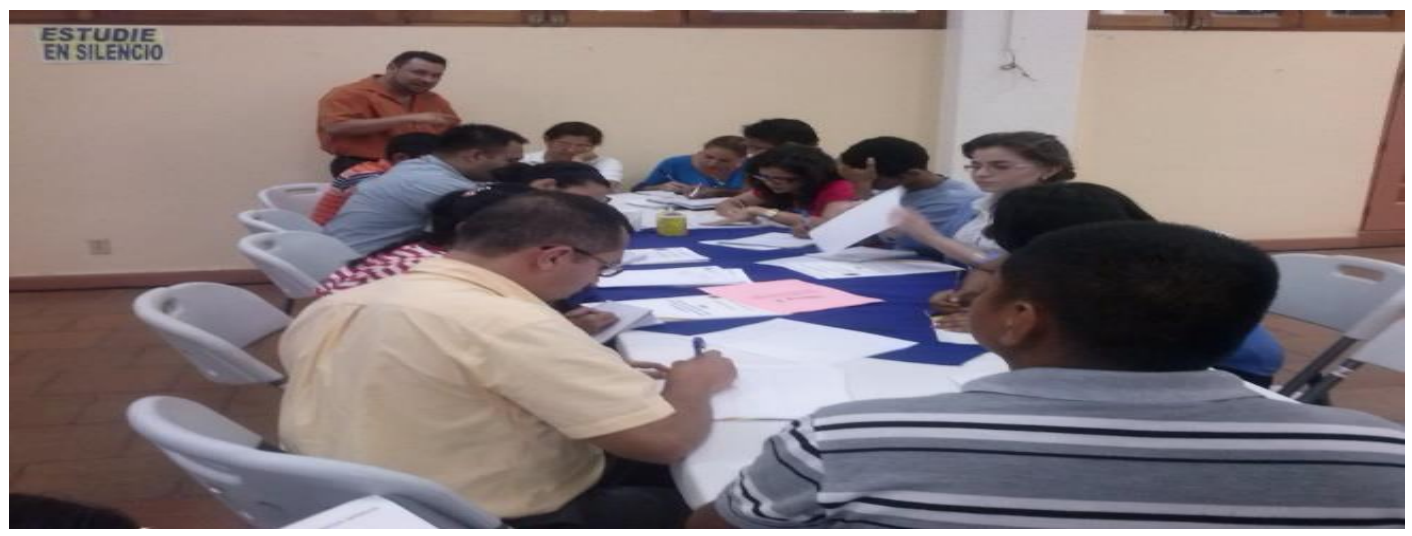

Figura 6. Coordinadores: MSc. Juan de Dios Bonilla/ MSc.Rubén Dormus

$\checkmark$ Mesa 4: Innovación en Industria Agroalimentaria

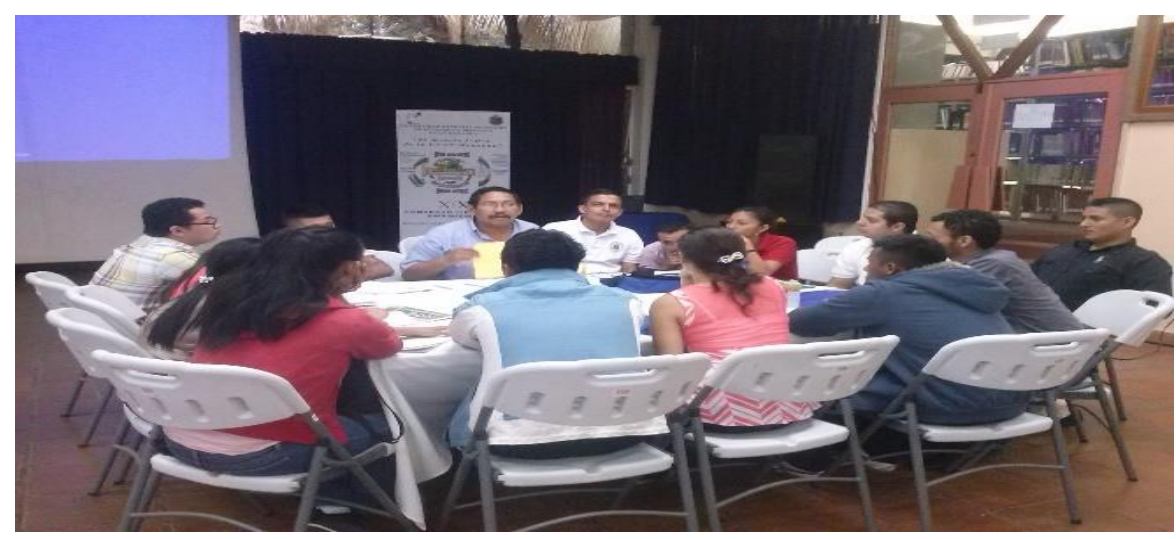

Figura 7. Coordinadores: M.A. Mario López/ Lic. Henry Murillo 
Mesa 5: Innovación Socio Educativo y Cultural

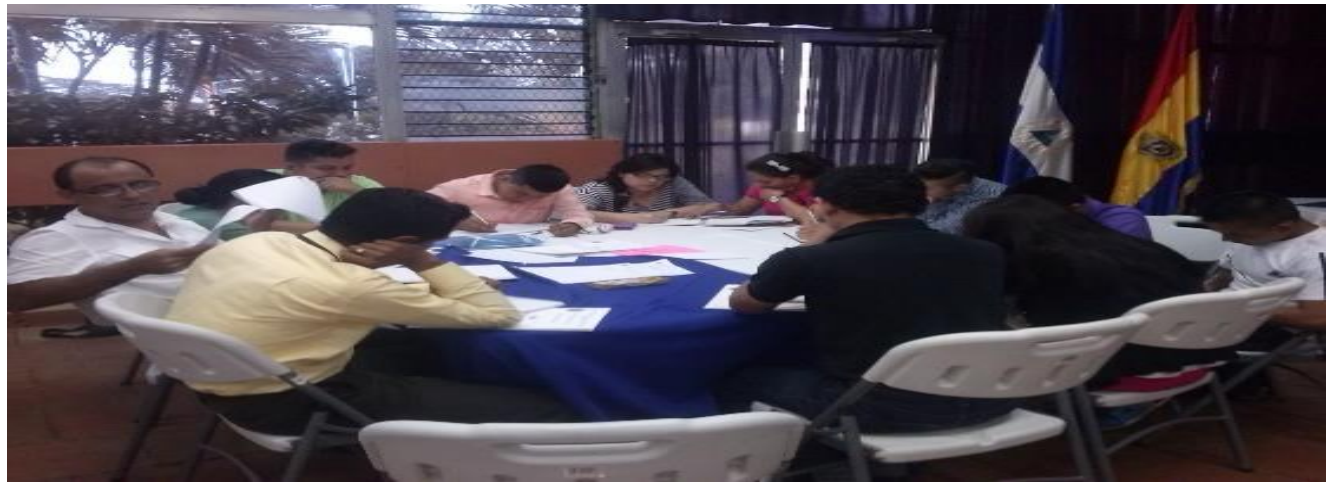

Figura 8. Coordinadores: MSc. Julio Orozco/ MSc. Ángela Gutiérrez

Los coordinadores de cada mesa de trabajo explicaron al grupo de estudiantes y docentes asignados, las problemáticas actuales u oportunidades existentes basados en la temática o línea de innovación correspondiente, luego los participantes iniciaron una lluvia de ideas,. Para identificar inconvenientes, Seguido llenaron el formato 1 "Diez Inconvenientes" donde identificaron 10 problemas u oportunidades de negocio a partir de la temática antes discutida, después seleccionaron por mesa de trabajo 5 problemas u oportunidades, seguido le dieron solución a esos problemas u oportunidades mediante productos innovadores en el formato 2," Priorización de oportunidades y soluciones". Posteriormente llenaron el formato 3 "Evaluación de idea" donde se evalúa las ideas con criterios tales como: tecnología, mercado, competencia etc. Finalmente se completa el Formato 4: Selección de ideas donde se seleccionaron dos productos o servicios innovadores y justificaron los beneficios y beneficiarios de esta nueva idea (Ver Formatos en Anexos)

Finalmente un miembro de cada mesa de trabajo expuso los resultados del ejercicio práctico realizado. (Productos o servicios nuevos).

\section{Resultados Obtenidos}

- Se obtuvieron 10 ideas de productos innovadores correspondientes a 5 líneas o temáticas de innovación.

- Se estableció el compromiso de que todos los estudiantes participantes en el taller de inducción participarán en la segunda convocatoria de proyectos de innovación de UNAN Managua, y los docentes se integrarán en calidad de asesores de esos proyectos. 
- Se motivó a los estudiantes y docentes participantes a aplicar a todas las convocatoria del CONICYT, destacándose los siguientes:

1. IX Edición del Premio Nacional a la Innovación

2. Convocatoria Reconocimiento a la trayectoria científica

3. Concurso Juvenil Aplicaciones Móviles

- Se promovieron la Red de Jóvenes Investigadores, Innovadores y Emprendedores de UNAN Managua y como la Red de Docentes Investigadores, Innovadores y Emprendedores de UNAN Managua.

- Se promovió en los estudiantes, para que estos desarrollen proyectos competitivos y que su impacto se refleje en su calidad profesional, esto se logró mediante la ponencia de experiencias de un joven innovador y emprendedor.

- Se reafirmó el compromiso en los estudiantes y docentes participantes, para que estos sean facilitadores en divulgar la información recibida en el taller y replicar en sus facultades los conocimientos adquiridos.

La comisión de Innovación cuenta con una base de más de 200 miembros entre estudiantes y docentes, que son promotores de la innovación y emprendimiento de la UNAN - Managua. 